\title{
A Multi-level Approach to Planning and Scheduling Resources for Aviation Training
}

\author{
Michael Johnstone ${ }^{a}$, Vu Le $^{\text {a }}$, Ana Novak ${ }^{\text {b }}$, Burhan Khan ${ }^{\text {a, Doug Creighton }}{ }^{\text {a }}, \underline{\text { Luke Tracey }}{ }^{\text {b }}$ \\ and Vivian Nguyen ${ }^{b}$ \\ ${ }^{a}$ Centre for Intelligent Systems Research, Deakin University, VIC, Australia \\ ${ }^{b}$ Defence Science and Technology Group, VIC, Australia \\ Email: luke.tracey@dsto.defence.gov.au
}

\begin{abstract}
This paper describes a multi-level system dynamics (SD) / discrete event simulation (DES) approach for assessing planning and scheduling problems within an aviation training continuum. The aviation training continuum is a complex system, consisting of multiple aviation schools interacting through interschool student and instructor flows that are affected by external triggers such as resource availability and the weather.
\end{abstract}

SD was used to model the overall training continuum at a macro level to ascertain relationships between system entities. SD also assisted in developing a shared understanding of the training continuum, which involves constructing the definitions of the training requirements, resources and policy objectives. An end-to-end model of the continuum is easy to relate to, while dynamic visualisation of system behaviour provides a method for exploration of the model.

DES was used for micro level exploration of an individual school within the training continuum to capture the physical aspects of the system including resource capacity requirements, bottlenecks and student waiting times. It was also used to model stochastic events such as weather and student availability. DES has the advantage of being able to represent system variability and accurately reflect the limitations imposed on a system by resource constraints.

Through sharing results between the models, we demonstrate a multi-level approach to the analysis of the overall continuum. The SD model provides the school's targeted demand to the DES model. The detailed DES model is able to assess schedules in the presence of resource constraints and variability and provide the expected capacity of a school to the high level SD model, subjected to constraints such as instructor availability or budgeted number of training systems. The SD model allows stakeholders to assess how policy and planning affect the continuum, both in the short and the long term.

The development of this approach permits moving the analysis of the continuum between SD and DES models as appropriate for given system entities, scales and tasks. The resultant model outcomes are propagated between the continuum and the detailed DES model, iteratively generating an assessment of the entire set of plans and schedule across the continuum. Combining data and information between SD and DES models and techniques assures relevance to the stakeholder needs and effective problem scoping and scaling that can also evolve with dynamic architecture and policy requirements.

An example case study shows the combined use of the two models and how they are used to evaluate a typical scenario where increased demand is placed on the training continuum. The multi-level approach provides a high level indication of training requirements to the model of the new training school, where the detailed model indicates the resources required to achieve those particular student levels.

Keywords: $\quad$ System dynamics, discrete event simulation, training continuum 
Johnstone, Le, Novak, Khan, Creighton, Tracey \& Nguyen. A Multi-level Approach to Planning and Scheduling Resources for Aviation Training

\section{INTRODUCTION}

The Australian Defence Force helicopter training continuum ${ }^{1}$ (HTC) is currently consolidating training schools and platforms across multiple services, with the rationalisation expected to provide a more efficient training continuum. Key to this process is the provision of a joint Helicopter Aviation Training School (HATS) to provide initial helicopter flight training with a large synthetic component. While managing the transition to HATS, it is important to understand dependencies within the HTC. Multi-level simulation provides the opportunity to explore relationships between entities and also relationships between constraints, costs and performance that span entities, providing insight to decision makers to assist in planning for the future state of the HTC.

This paper presents a multi-level approach to planning and scheduling for the future state of the HTC. We present a system dynamics (SD) model of the end-to-end continuum flows, complemented by a micro level discrete event simulation (DES) model of the new HATS facility.

The SD model has been developed with stakeholders, following the iterative design and incremental development approach, to facilitate a shared understanding of the continuum and associated flows, dependencies and feedback loops. This macro level approach provides insight as to the required detail within the DES model, and where to define model boundaries. Additionally the model allows the exploration of dynamic behaviour within the HTC.

The DES model considers physical aspects of the new HATS facility, with a focus on resource capacity requirements, bottlenecks, student queues and associated waiting times. When combined with stochastic events such as student availability and bad weather, the DES model is able to predict the ability of the school to meet scheduling requirements and provide an indication of student throughput, queues, in-system times and resource utilization performance metrics.

Through iterative and alternating simulation model runs, data and information is shared between the models and is used to update model parameters or fully explore how operational policies will be reflected in the future state of the HTC. The DES model provides an indication of the ability of the new HATS facility to meet a particular policy in terms of student targets and instructor availability as defined by the SD model with the constraints of available mission systems and in the presence of variable weather and student availability. The results from the DES model provides an input back into the SD model, where the impacts of the new school are able to be explored.

This paper begins by exploring existing literature that provides the foundation for multiscale models utilising $\mathrm{SD}$ and DES. Next, a description of the problem is given, followed by a discussion on what role SD and DES plays in this research. Subsequently, our method used for integrating SD and DES for the HTC is presented. A case study using the technique will then be explored. Finally, a concluding remark on the findings will be given.

\section{RELATED LITERATURE}

System dynamics attempts to discover the behaviour over time of complex systems (Forrester, 1995). SD can visualise a system through a qualitative causal loop diagram, or provide quantitative analysis through a stock and flow simulation model, both methods providing an understanding of behaviour to better prepare decision makers when targeting specific system outcomes. Often used at a strategic level to adjust system behaviour through targeted system intervention, SD is an applicable methodology for problem conceptualisation, recognition and understanding (Coyle, Exelby, \& Holt, 1999; Wolstenholme, 1983), with collaborative potential through group model building (Nahavandi, Creighton, Le, Johnstone, \& Zhang, 2015; Vennix, 1999).

Previous applications of SD to military training have shown the utility of the method to identify and provide an understanding of relationships within the system and to allow stakeholders to conceptualise the system (Armenia, Centra, Cesarotti, De Angelis, \& Retrosi, 2012; Thomas, Kwinn, McGinnis, Bowman, \& Entner, 1997; Wang, 2005). When applied to training schools, the effects of dynamic demand and resource constraints have resulted in the identification of student queues and delays incurred (Dinges, 2009; Enos, 2011).

Discrete event simulation provides the ability to model a system with a low level of detail and is well suited to model stochastic, queuing systems (Creighton, Johnstone, Le, Nahavandi, \& Zhang, 2013; Johnstone,

\footnotetext{
${ }^{1}$ A training continuum encapsulates individual and collective training activities that together prepare students, undertaking the training, for deployment to operational squadrons.
} 
Johnstone, Le, Novak, Khan, Creighton, Tracey \& Nguyen. A Multi-level Approach to Planning and Scheduling Resources for Aviation Training

Creighton, \& Nahavandi, 2015; Zhang, Le, Johnstone, Nahavandi, \& Creighton, 2012). DES is able to incorporate physical elements and attributes of the system, providing a high fidelity abstraction of the original system, resulting in a model that can be used for tactical and operational prediction and analysis.

Previously, DES has been employed to study waiting times at a military training school (Davenport, Neu, Smith, \& Heath, 2007). Simulation results for scenarios comparing alternate scheduling methods, showed the utility of DES to explore and evaluate system changes. DES has also been used to evaluate schedule feasibility and risk (Le et al., 2015). Here DES provided feedback to planners on the effectiveness of their production schedules, allowing for the iterative improvement of schedules to mitigate risk.

Architectures to support the combination of SD \& DES have previously been proposed (Chahal \& Eldabi, 2008). Extensive work in healthcare simulation has looked into the effectiveness of merging the two paradigms (Brailsford, Desai, \& Viana, 2010; Brailsford, Viana, Rossiter, Channon, \& Lotery, 2013; Heath, Buss, Brailsford, \& Macal, 2011), where it was discussed that the effort to integrate modelling paradigms in one platform may outweigh the benefits. Simpler architectures, such as stand-alone models sharing data (Chahal \& Eldabi, 2008), may sufficiently meet the needs of the simulation study.

These findings have been considered in forming our approach. Here stand-alone models are used with a mechanism for sharing data. This enables a more efficient distribution of workload and utilisation of skills within the team, without the need to develop an integrated platform, or to learn a new package where the integration has been incorporated. Additionally, the package used does not necessarily add value to the stakeholder, as it is the results from the method chosen that are important.

\section{THE TRAINING CONTINUUM}

The HTC considered in this paper consists of four squadrons and eight training schools, see Figure 1, where six student types and three instructor types transition through the continuum. The path through the continuum is set for each student type, with a typical length of four years. Instructors are drawn from squadrons if capacity allows, to each school as required. The return on the training investment is made through service time by students in the operational squadrons, therefore it is of interest to reduce the training time within the HTC so as to maximize the operational service time.

Student progression is affected by failure rates at each school, and constrained due to instructor availability, the school's capacity and a school's targeted number of graduates. Instructor postings are for a period of two years, after which they either return to the squadron or move to a subsequent school.

The HTC is affected by school policies, class sizes, pass rates, plus changes to operational squadron requirements and platforms. Without a system wide view, it is difficult to assess how changes will propagate through the HTC over time.

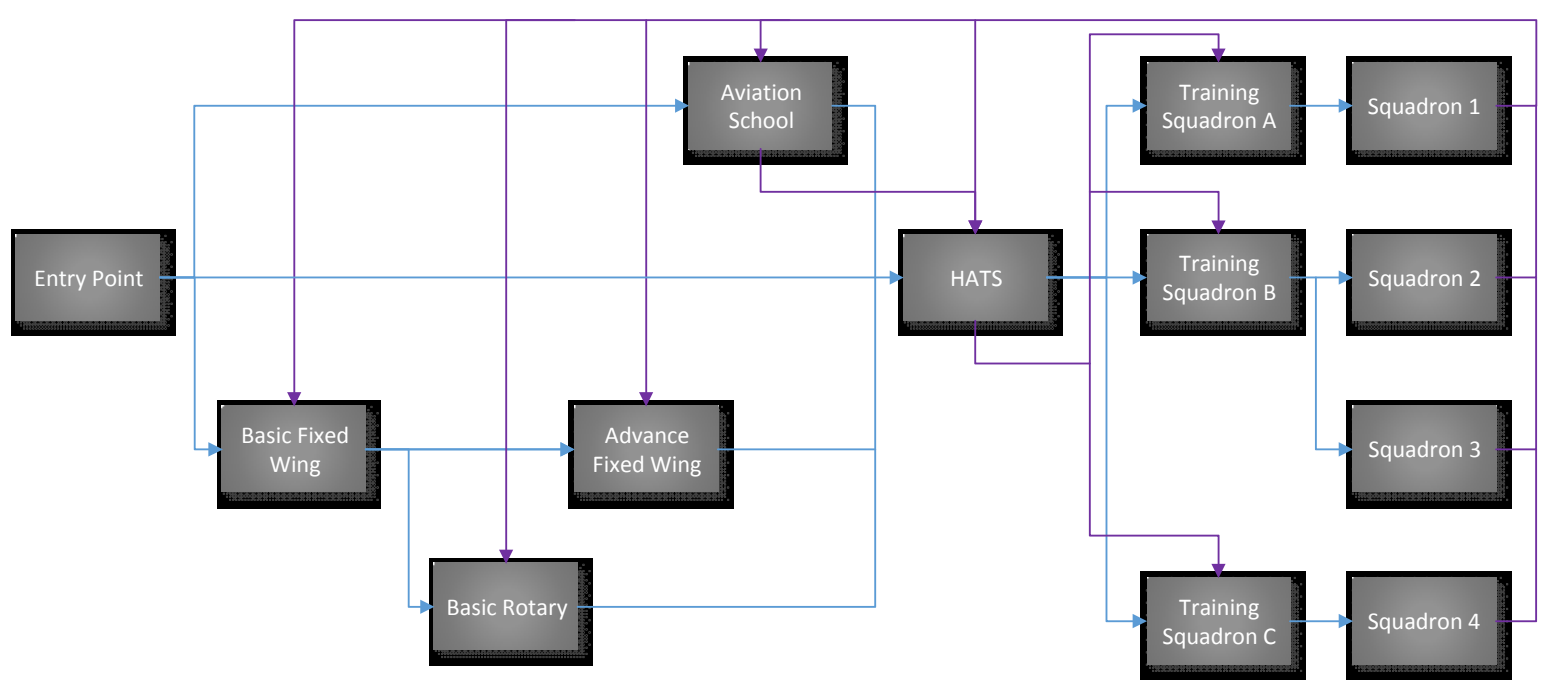

Figure 1. The helicopter training continuum, with student flows represented by blue lines and instructor flows represented by purple lines. The feedback of squadrons supplying instructors to train students is evident. 
Johnstone, Le, Novak, Khan, Creighton, Tracey \& Nguyen. A Multi-level Approach to Planning and Scheduling Resources for Aviation Training

\section{SD MODEL}

SD is utilised to provide a macro level view of the end-to-end continuum flows. The continuum is encoded as a stock and flow model that is able to reproduce the dynamic behaviour of the HTC and provide a visualisation of the behaviour and relationships between variables. Within the model, the six student and three instructor types are considered as stocks that flow between schools. The flow rates are determined using a combination of instructor availability, school capacity and student availability, which all aim to push students through the HTC. The squadrons annual targets are combined with school pass rates to set targets for each school and can also be used to influence the student flow.

Each squadron consists of a different mix of student types, matching the respective platform of the squadron, and has a workforce target that is required to be maintained in order to remain operationally ready. Squadrons lose staff through an attrition rate, therefore the required intake to the squadron is able to be calculated. As it takes four years for a student to complete training, planning is essential to ensure there is neither an oversupply nor undersupply to each squadron.

Additionally, each squadron is responsible for providing instructors to the HTC to train students. Each school's planned training volume sets the demand for instructors in that school. This system level feedback is of particular interest. To increase a squadron's workforce the HTC increases student numbers, which increases instructor demand, which reduces a squadron's workforce in the short term.

To provide both stakeholders and modellers with a unified view of the HTC, the SD model was developed incrementally with stakeholder feedback gathered during interactive model demonstration workshops. Figure 2 presents the relationships between variables that influence the rate of students entering a school.

The intention of the SD model is to provide at a high level representation of how the continuum will behave, with a focus on the dynamic behaviour of relationships between variables. Policy changes or other disturbances are injected into the model, which then attempts to reach a new steady state. The period of time to reach the new steady state, and the relative change to the previous steady state, serve as a communication mechanism for engaging stakeholders. The model can support the identification and planning of risk mitigation strategies through the modification of levers within the model and observation of the impacts to the continuum as they propagate over time. For example, an increased demand for pilots at an operational squadron translates to an increased intake of students at the entry point training school. Too large an intake could potentially create a bottleneck in the system, while a low intake might not meet the demand. The SD model, through its levers, allows the stakeholders to derive a more feasible intake amount, and thus reduce the associated risk.

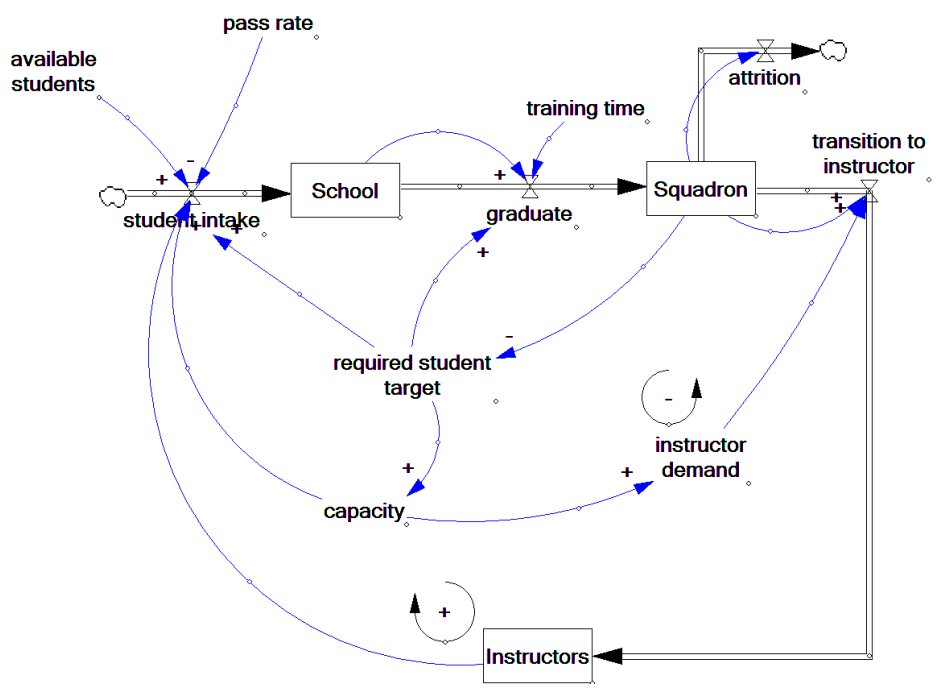

Figure 2. A snapshot of the SD model showing selected variables that influence student intake into a school.

\section{DES MODEL}

While the SD model provides a representation of the dynamic behaviour of the HTC, there remains a large unknown regarding the performance of the new HATS facility, especially in the presence of operational variability. To inform the SD model of required student target rates for HATS, the knowledge regarding pass 
Johnstone, Le, Novak, Khan, Creighton, Tracey \& Nguyen. A Multi-level Approach to Planning and Scheduling Resources for Aviation Training

rates from existing similar schools can be used as an estimate. To assess the ability of HATS to meet these targets requires a different approach.

The results from the winning bid of a competitive tendering process for the design and operation of HATS are used to construct a DES model of the proposed facility. Information from the bid informs the model construction, including training aircraft and mission systems, instructor levels and student training programs. DES model results are used to assess if the combination of resources and training schedules are achievable in the presence of stochastic events such as student availability and bad weather.

The approach taken for control within the DES model is to use the training programs to drive day-to-day events, as per Figure 3. Each student type has their own program that defines for each day the class, instructor and resources required to complete the training activity. Each day, students reserve instructors and resources, perform their training activity, and then release the reserved items. If an activity is not completed due to a lack of resources or instructors, bad weather or student unavailability, then a student attempts to catch up on a free day within their program.

Over repeated runs, DES generates a statistical estimate of the ability of the HATS facility to meet the requirements as set by the school design specifications and constraints imposed by the SD model. This is expressed in terms of both graduated students in the presence of limited instructors and resources, and variability due to student availability and weather.

This approach uses DES to provide low-level modelling resolution at the class and resource level, providing the advantage of being able to determine resource requirements and the impact in terms of cost of this decision. Also, a DES methodology facilitates bottleneck identification, resource utilisation, and the ability to understand the impact of variability on the system.

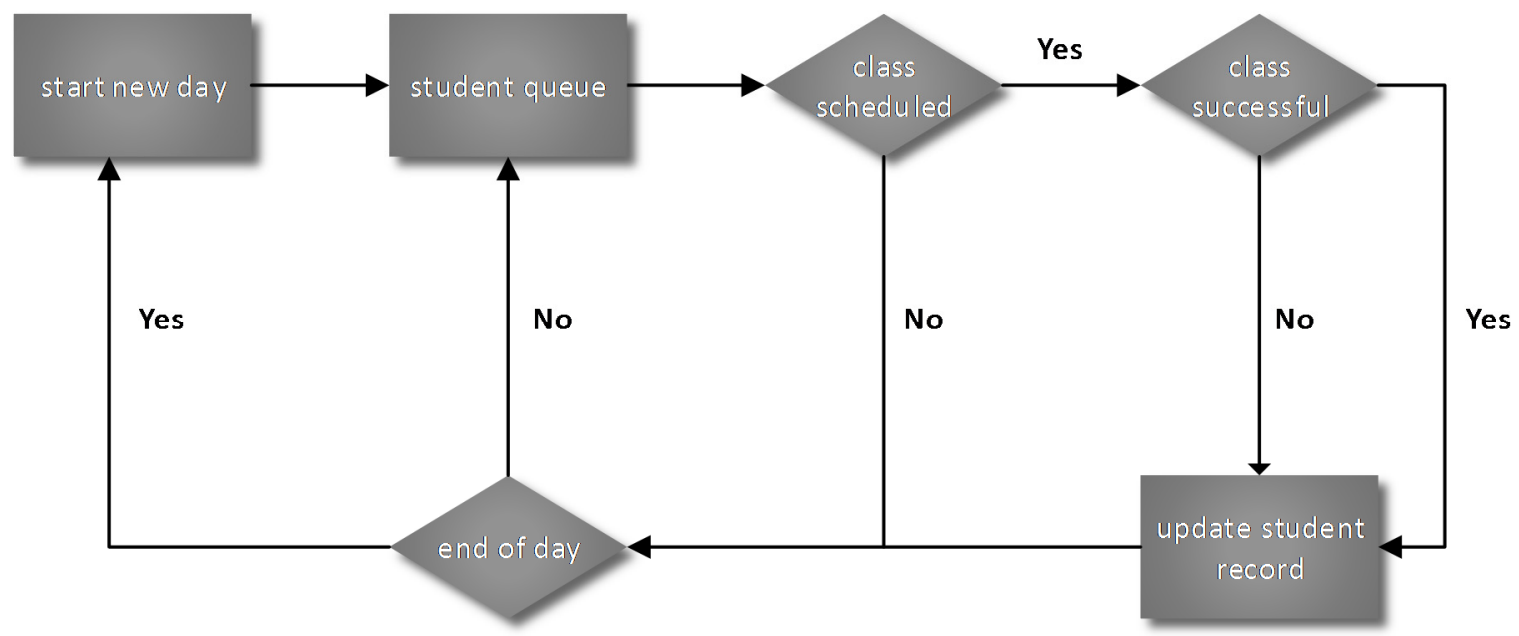

Figure 3. High level representation of the control logic for the DES model, Students queue at the start of the day and commence training subject to resource availability. Post training they either return to this queue or wait for the next day to begin.

\section{A MULTI-LEVEL APPROACH FOR THE MULTI-LEVEL MODELS}

The developed SD model visualises the dynamic behaviour of the HTC, assisting the HTC planning process by representing relationships and behaviour, and provides a tool to test various scenarios and observe impacts to the HTC varying resource capacities, constraints and operating conditions.

The SD model provides an indication of the required number of graduates expected from each school. This information is used by the DES model to determine a) the level of resources required to meet that demand and b) if those resources are constrained, the performance the school will be able to achieve in the presence of stochastic events.

The knowledge gained from the DES model can then be used within the SD model to show the impact on the schools ability to meet demand within the continuum, and how this plays out in the future as the impacts ripple across the continuum, as depicted in Figure 4. It is the visibility of these long term effects that are of particular interest to stakeholders. For example, reducing the number of training aircraft may only slightly increase the 
Johnstone, Le, Novak, Khan, Creighton, Tracey \& Nguyen. A Multi-level Approach to Planning and Scheduling Resources for Aviation Training

duration of students within a school. However, the additional delay may impact on the ability of downstream schools to meet their targets several years into the future.

Currently a detailed DES model exists for a single school. The modelling approach taken will quickly allow for the integration of models of subsequent schools as the need arises. By combining knowledge between the models, stakeholder needs, which are often dynamic, are satisfied as the approach assures relevance. In discussions with stakeholders, scenarios are proposed at either an end-to-end continuum level, or within a school DES model. As the scenario plays out, either model may be updated to assess impacts. If the need arises to create a more detailed model of another school, this model can be integrated into the existing process in a similar way via the common data interface and data sharing mechanism.

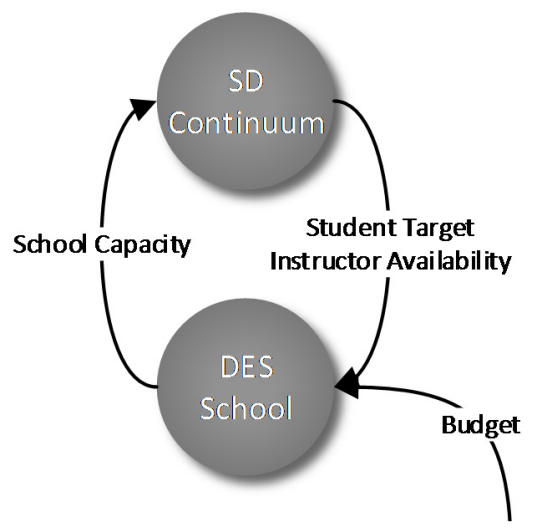

Figure 4. The relationship between the SD and DES models. Here student targets and instructor availability is provided to the DES model, which can determine school capacity subject to budget constraints.

\section{CASE STUDY}

Here we present a scenario to demonstrate the proposed approach. The SD model is initialised into the current steady state and the required number of pilots for Squadron 1 is increased by $50 \%$. The SD model calculates the propagated increase of student intake/requirement to achieve the new Squadron 1 target, as per Table 1. The DES model is then engaged to evaluate whether HATS has sufficient capacity to support the new student targets and instructor availability, as calculated by the SD model. The procedure considers measures of effectiveness such as length of the course, missed training activities and queueing behaviour. As seen in Table 1, the SD predicts that HATS will need to increase the student intake from 14 to 19 in the first year, and in order to achieve this it will require an additional 3 instructors. However, the DES model shows that the implication of the increased number of students was a bottleneck in the full flight simulator (FFS) component of the training. The consequence being prolonged length of training in this school, which compromises the efficiency of the finely tuned training continuum. In order to reach appropriate level of capacity to support the new student throughput an additional simulator would need to be purchased.

Table 1. The yearly expected demand on the HATS school and the requirements calculated to meet these targets.

\begin{tabular}{|r|r|r|c|}
\hline \multicolumn{1}{|c|}{ Year } & \multicolumn{1}{|c|}{ Pilots Target } & Instructors Count & DES Capacity \\
\hline 0 & 14 & 15 & - \\
\hline 1 & 19 & 18 & $+1 \mathrm{FFS}$ \\
\hline 2 & 19 & 17 & $+1 \mathrm{FFS}$ \\
\hline 3 & 18 & 16 & $+1 \mathrm{FFS}$ \\
\hline 4 & 19 & 17 & $+1 \mathrm{FFS}$ \\
\hline
\end{tabular}

\section{CONCLUSION}

This paper presents an approach to modelling a training continuum at a high level, which is able to drill down when required, to provide a higher fidelity estimate of the performance of individual schools within the continuum. SD provided a method to engage stakeholders, understand the system and problem and visualise 
Johnstone, Le, Novak, Khan, Creighton, Tracey \& Nguyen. A Multi-level Approach to Planning and Scheduling Resources for Aviation Training

the behaviour of the system over time. SD was unable to quantify the performance of individual schools within the continuum in the presence of resource constraints and stochastic events. For these situations we used DES to provide the metrics to the SD model. This approach of moving between SD and DES combined data and information between the multi-level models and maintained relevance to the stakeholder needs and effective problem scoping and scaling that can also evolve with dynamic requirements.

The multi-level approach presented in this paper is generalisable to other defence and civilian training continuums.

\section{REFERENCES}

Armenia, S., Centra, A., Cesarotti, V., De Angelis, A., \& Retrosi, C. (2012). Military Workforce Dynamics and Planning in the Italian AirForce. Paper presented at the 30th International SD Conference.

Brailsford, S. C., Desai, S. M., \& Viana, J. (2010). Towards the holy grail: combining system dynamics and discrete-event simulation in healthcare. Paper presented at the Proceedings of the 2010 Winter Simulation Conference.

Brailsford, S. C., Viana, J., Rossiter, S., Channon, A., \& Lotery, A. J. (2013). Hybrid simulation for health and social care: The way forward, or more trouble than it's worth? Paper presented at the Proceedings of the 2013 Winter Simulation Conference.

Chahal, K., \& Eldabi, T. (2008). Applicability of hybrid simulation to different modes of governance in UK healthcare. Paper presented at the Simulation Conference, 2008. WSC 2008. Winter.

Coyle, J., Exelby, D., \& Holt, J. (1999). System dynamics in defence analysis: some case studies. Journal of the Operational Research Society, 372-382.

Creighton, D., Johnstone, M., Le, V., Nahavandi, S., \& Zhang, J. (2013). Simulation-based operational decision support systems. Paper presented at the 20th International Congress on Modelling and Simulation.

Davenport, J., Neu, C., Smith, W., \& Heath, S. (2007). Using discrete event simulation to examine marine training at the Marine Corps Communication-Electronics school. Paper presented at the Proceedings of the 2007 Winter Simulation Conference.

Dinges, J. (2009). Army Aviation Training Program: Interim Analysis Review Board. Center For Army Analysis. Washington DC (as cited in Enos, 2011).

Enos, J. R. (2011). Dynamics of Combat Aviator Training. Paper presented at the Proceedings of the 29th International Conference of the System Dynamics Society.

Forrester, J. W. (1995). The beginning of system dynamics. McKinsey Quarterly, 4-17.

Heath, S. K., Buss, A., Brailsford, S. C., \& Macal, C. M. (2011). Cross-paradigm simulation modeling: challenges and successes. Paper presented at the Proceedings of the 2011 Winter Simulation Conference.

Johnstone, M., Creighton, D., \& Nahavandi, S. (2015). Simulation-based baggage handling system merge analysis. Simulation Modelling Practice and Theory, 53, 45-59.

Le, V. T., Johnstone, M., Zhang, J., Khan, B., Creighton, D., Hanoun, S., \& Nahavandi, S. (2015). Complex Simulation of Stockyard Mining Operations Advances in Global Optimization (pp. 529-537): Springer International Publishing.

Nahavandi, S., Creighton, D., Le, V. T., Johnstone, M., \& Zhang, J. (2015). Future Integrated Factories: A System of Systems Engineering Perspective. In M. Fathi (Ed.), Integrated Systems: Innovations and Applications (pp. 147-161): Springer International Publishing.

Thomas, D., Kwinn, B. T., McGinnis, M., Bowman, B., \& Entner, M. D. (1997). The US army enlisted personnel system: a system dynamics approach. Paper presented at the IEEE International Conference on Systems, Man, and Cybernetics.

Vennix, J. A. (1999). Group model-building: tackling messy problems. System Dynamics Review, 15(4), 379401.

Wang, J. (2005). A Review of Operations Research Applications in Workforce Planning and Potential Modeling of Military Training: DTIC Document.

Wolstenholme, E. (1983). System dynamics: A system methodology or a system modelling technique. Dynamica, 9, 84-90.

Zhang, J., Le, V., Johnstone, M., Nahavandi, S., \& Creighton, D. (2012). Discrete Event Simulation Enabled High Level Emulation of a Distribution Centre. Paper presented at the 2012 UKSim 14th International Conference on Computer Modelling and Simulation (UKSim). 